\title{
Mass spectral compatibility of four proteomics stains
}

\author{
Malcolm S. Ball1 ${ }^{1}$, and Peter Karuso ${ }^{1,2 *}$. \\ ${ }^{1}$ FLUOROtechnics Pty Ltd, Macquarie University, Sydney, NSW, 2109, Australia. \\ ${ }^{2}$ Department of Chemistry \& Biomolecular Sciences, Macquarie University, Sydney, NSW, \\ 2109, Australia.
}

peter.karuso@mq.edu.au

\section{SUPPORTING INFORMATION}

* Correspondence: Professor Peter Karuso, Department of Chemistry \& Biomolecular Sciences, Macquarie University, Sydney, NSW, 2109, Australia. Phone: +61298508290; Fax: +61298508313; e-mail: peter.karuso@mq.edu.au

\section{Gel Images.}

A copy of every gel image used in this research is presented as Figure S-1. Gels were stained as per the recommended protocols for optimum sensitivity rather than optimal speed. Gels were imaged on Typhoon 9200 (GE-Healthcare) using $532 \mathrm{~nm}$ laser and filters recommended by suppliers of the stains.

PMT settings were used for optimum spot/background characteristics as experimentally determined: Krypton, LavaPurple and SYPRO Ruby $=540 \mathrm{~V}$; Flamingo $=400 \mathrm{~V}$ but with identical brightness and contrast settings.

Images were exported to tagged image format (compressed TIF) without further modification. 

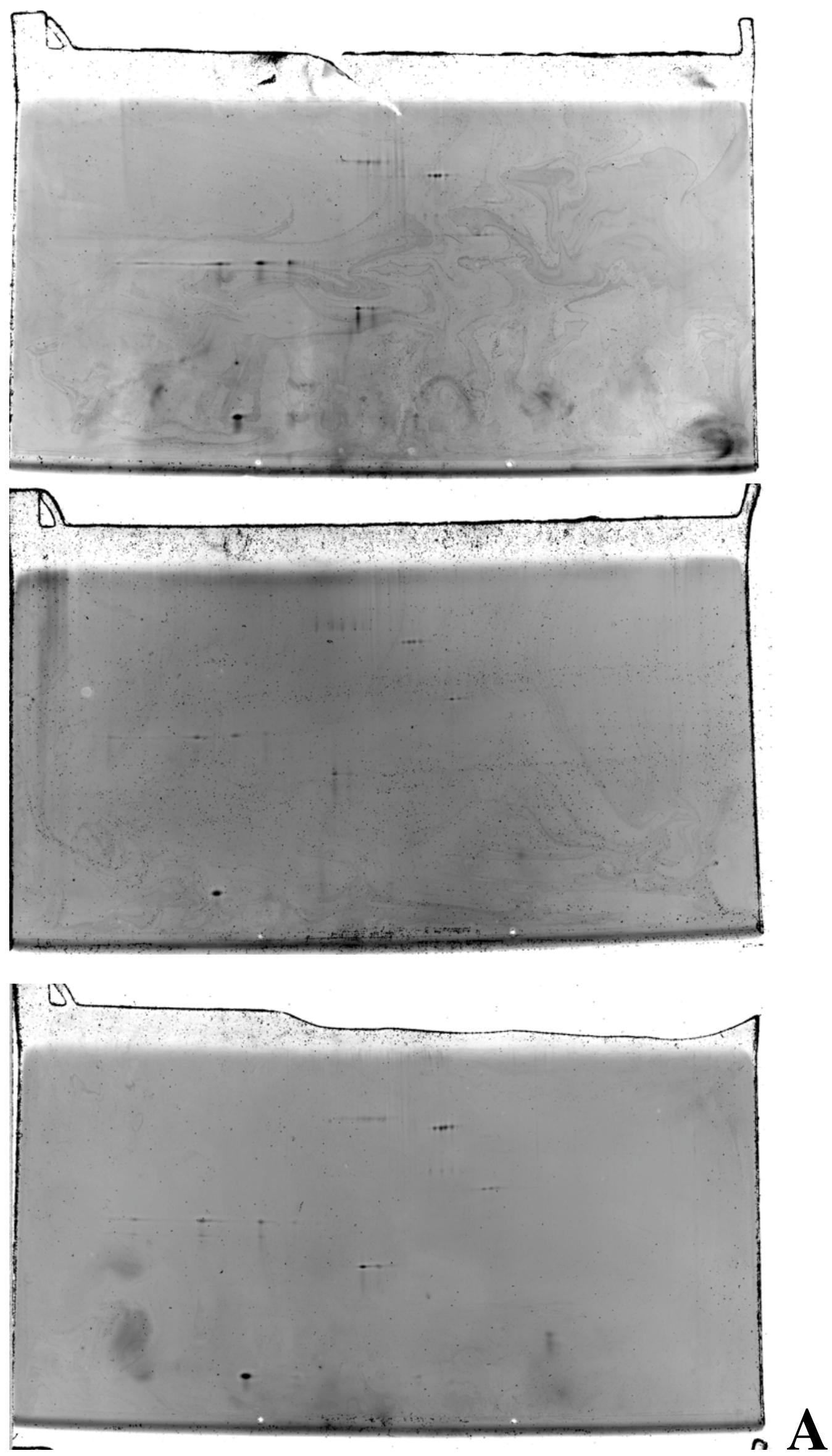

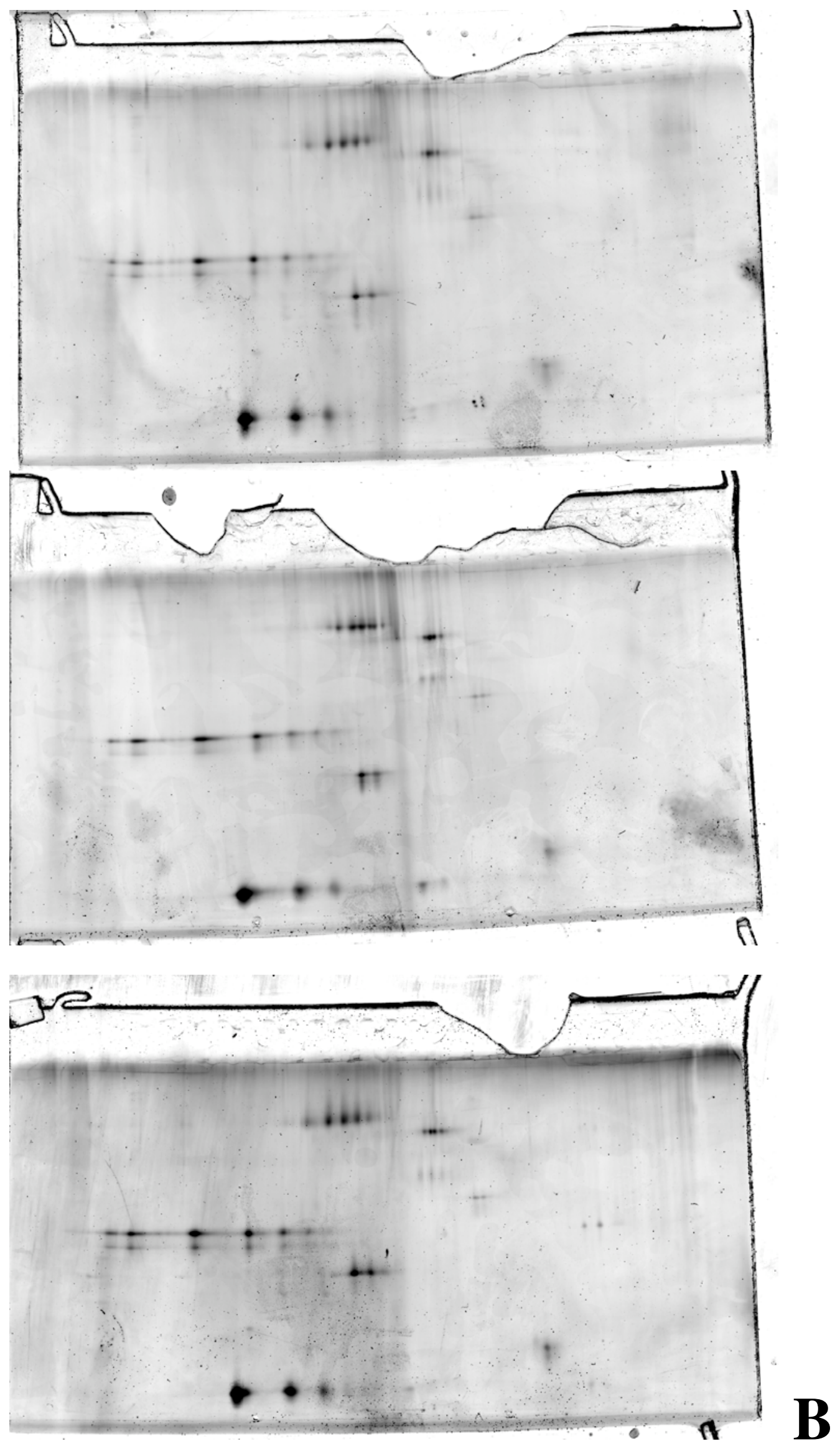


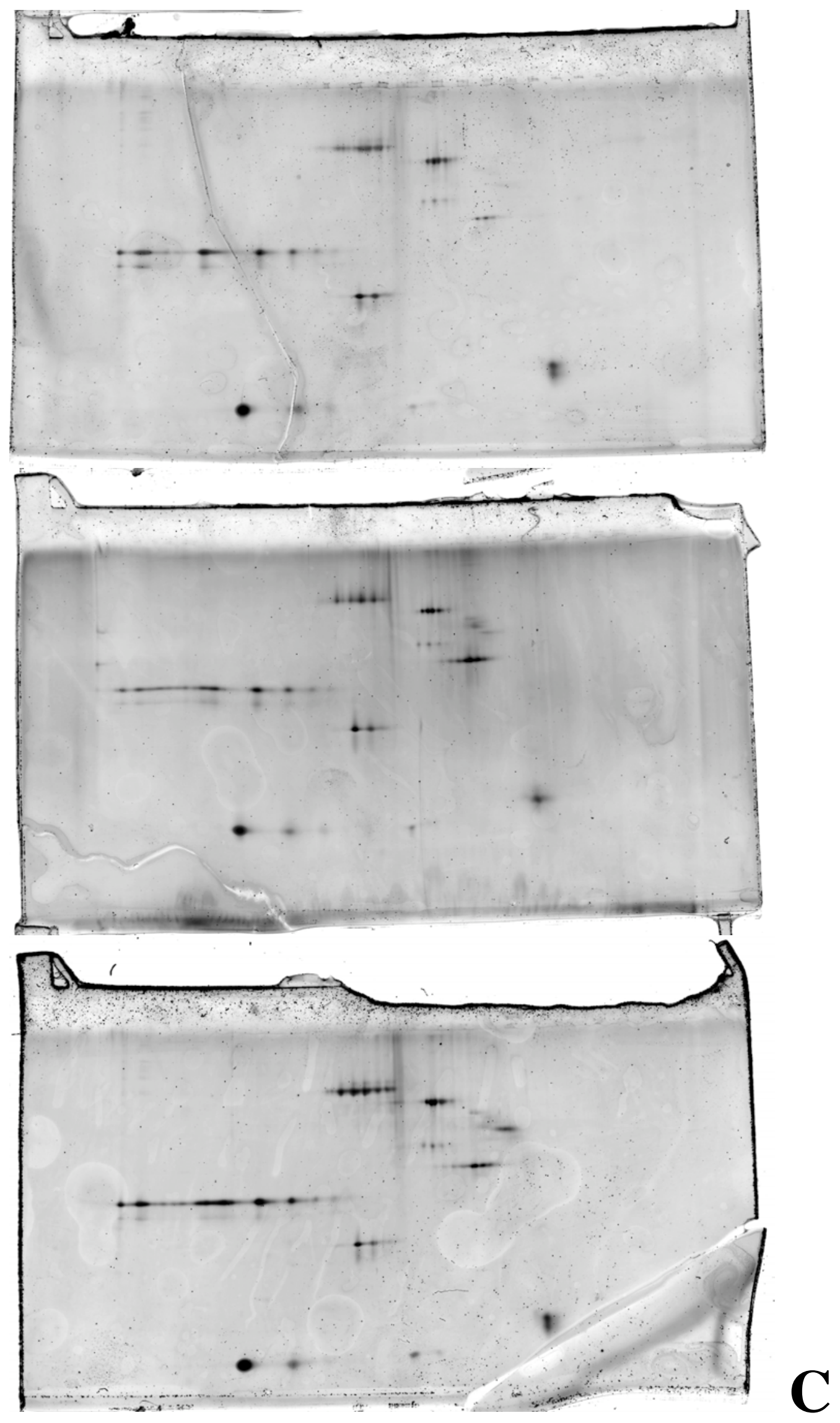




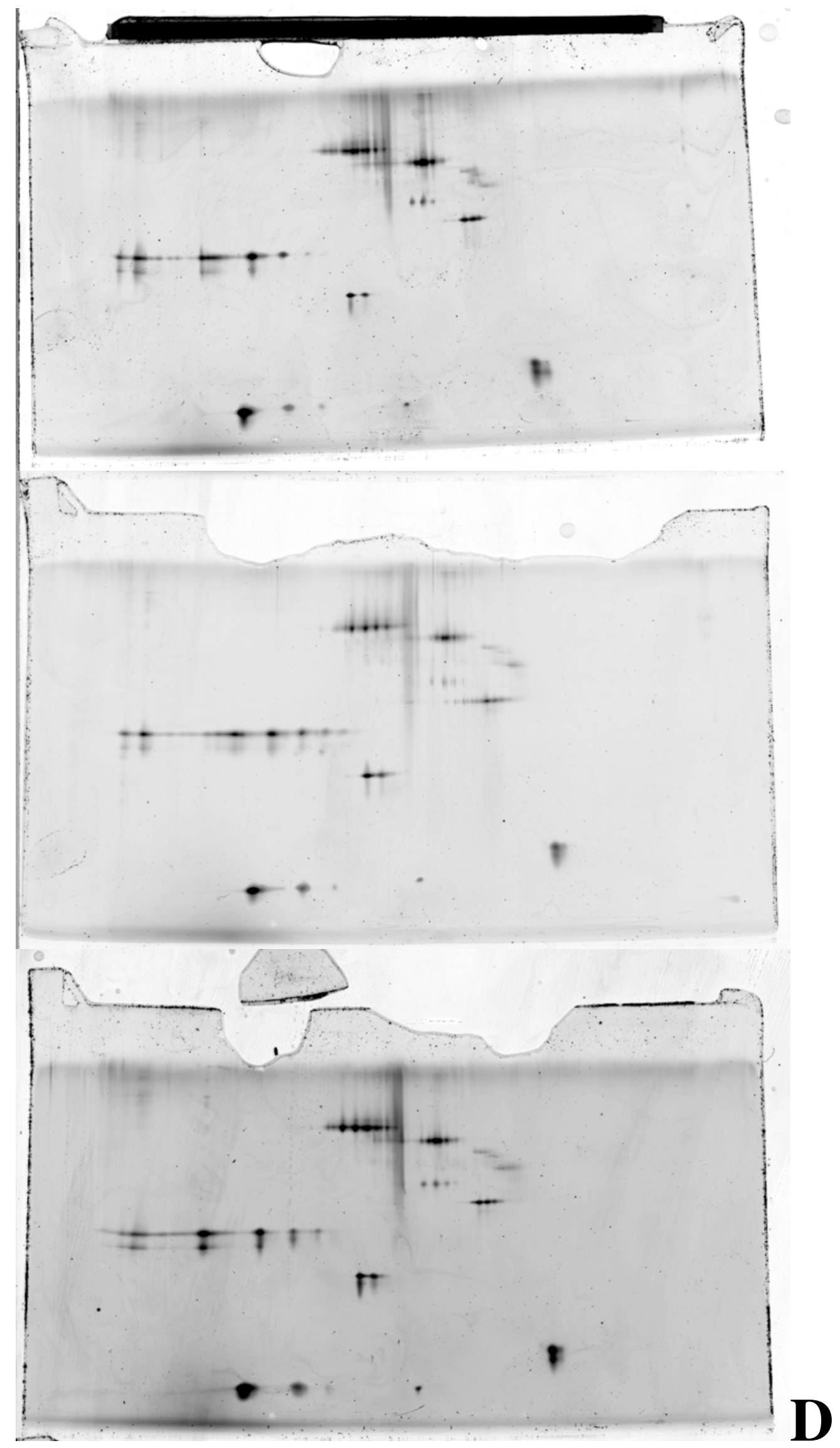


Figure S-1. Triplicate 2D PAGE markers (BioRad) separated on Criterian (BioRad) 8-16\% gel and stained with SYPRO Ruby (A), LavaPurple (B), Flamingo (C) and Krypton (D) total protein stains according to the suppliers' recommended protocols.

\section{Mass Spectral Data.}

Spots were manually cut from 2 gels of each stain using a clean pipette tip (to ensure that gel plug size was consistent between the stains). Gel plugs were placed into low protein binding 96 well plate and submitted for analysis at the Australian Proteome Analysis Facility (APAF) who performed the tryptic digestions and MALDI-ToF spectrometry without any knowledge of the sample origin. The results obtained are presented in Table S-1.

Full MASCOT database search results are available from the authors (1100 pages) upon request.

Plate 1:

B5-C12 = SYPRO Ruby stained gel plugs

SYPRO Ruby gel 1

B5-B6 = conalbumin

B7-B9 = bovine serum albumin (BSA)

B10-B11 = GADPH

$\mathrm{B} 12=$ myoglobin

$\mathrm{C} 1-\mathrm{C} 2=$ actin

SYPRO Ruby gel 2

$\mathrm{C} 3-\mathrm{C} 4=$ conalbumin

$\mathrm{C} 5-\mathrm{C} 7=\mathrm{BSA}$

$\mathrm{C} 8-\mathrm{C} 9=\mathrm{GADPH}$

$\mathrm{C} 10=$ myoglobin

C11-C12 = actin

D1-E8 LavaPurple stained gel plugs

LavaPurple gel 1

D1-D2 = conalbumin

D3-D4 = bovine serum albumin (BSA)

D5-D6 = GADPH

D7 = carbonic anhydrase

D8 = myoglobin

D9 $=$ actin

D10 = soybean trypsin inhibitor $(\mathrm{STI})$

LavaPurple gel 2

D11-D12 = conalbumin

$\mathrm{E} 1-\mathrm{E} 2=\mathrm{BSA}$

$\mathrm{E} 3-\mathrm{E} 4=\mathrm{GADPH}$

$\mathrm{E} 5=$ carbonic anhydrase

$\mathrm{E} 6=$ myoglobin

$\mathrm{E} 7=$ actin

$\mathrm{E} 8=\mathrm{STI}$

E9-G4 Flamingo stained gel plugs
Flamingo gel 1

E9-E10 = conalbumin

E11-E12 = bovine serum albumin (BSA)

$\mathrm{F} 1-\mathrm{F} 2=\mathrm{GADPH}$

$\mathrm{F} 3$ = carbonic anhydrase

$\mathrm{F} 4=$ myoglobin

F5 $=$ actin

D10 = STI

Flamingo gel 2

$\mathrm{F} 7-\mathrm{F} 8=$ conalbumin

$\mathrm{F} 9-\mathrm{F} 10=\mathrm{BSA}$

$\mathrm{F} 11-\mathrm{F} 12=\mathrm{GADPH}$

$\mathrm{G} 1=$ carbonic anhydrase

$\mathrm{G} 2$ = myoglobin

$\mathrm{G} 3=\operatorname{actin}$

$\mathrm{G} 4=\mathrm{STI}$

G5-H12 Krypton stained gel plugs

Krypton gel 1

G5-G6 = conalbumin

G7-G8 = bovine serum albumin (BSA)

G9-G10 = GADPH

G11 = carbonic anhydrase

G12 = myoglobin

$\mathrm{H} 1=\operatorname{actin}$

$\mathrm{H} 2=\mathrm{STI}$

Krypton gel 2

$\mathrm{H} 3-\mathrm{H} 4$ = conalbumin

$\mathrm{H} 5-\mathrm{H} 6=\mathrm{BSA}$

$\mathrm{H} 7-\mathrm{H} 8=\mathrm{GADPH}$

H9 = carbonic anhydrase

$\mathrm{H} 10=$ myoglobin

$\mathrm{H} 11=$ actin

$\mathrm{H} 12=\mathrm{STI}$ 
Table S-1. PMF results for gels stained with SYPRO Ruby, LavaPurple, Flamingo and Krypton.

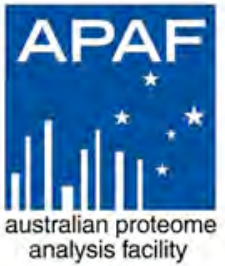

\begin{tabular}{|c|c|c|c|c|c|}
\hline Sample & Protein & $\begin{array}{l}\text { Sequence } \\
\text { coverage }\end{array}$ & $\begin{array}{l}\text { Mowse } \\
\text { Score }\end{array}$ & $\begin{array}{c}\text { Number } \\
\text { of } \\
\text { Peptides }\end{array}$ & $\begin{array}{c}\text { Missed } \\
\text { Cleavages }\end{array}$ \\
\hline A1 & alpha 2 macroglobulin [Homo sapiens] & $24 \%$ & 119 & 29 & 1 \\
\hline A2 & No Significant hit & & & & \\
\hline A3 & No Significant hit & & & & \\
\hline A4 & No Significant hit & & & & \\
\hline A5 & $\begin{array}{l}\text { Alpha-2-macroglobulin precursor } \\
\text { (Alpha-2-M) }\end{array}$ & $32 \%$ & 202 & 37 & 2 \\
\hline A6 & $\begin{array}{l}\text { Glucose Oxidase (non significant } \\
\text { match) }\end{array}$ & $27 \%$ & 71 & 12 & 1 \\
\hline A7 & $\begin{array}{l}\text { alpha-1 acid glycoprotein } \quad \text { [Bos } \\
\text { taurus] }\end{array}$ & & 70 & 9 & 4 \\
\hline A8 & Chain A, Crystal Structure of Avidin & $78 \%$ & 104 & 15 & 6 \\
\hline A9 & $\begin{array}{l}\text { Alpha-2-macroglobulin precursor } \\
\text { (Alpha-2-M) }\end{array}$ & $41 \%$ & 169 & 37 & 2 \\
\hline A10 & Chain, Glucose Oxidase (E.C.1.1.3.4 & $50 \%$ & 101 & 22 & 1 \\
\hline A11 & $\begin{array}{l}\text { alpha-1 acid glycoprotein } \quad \text { [Bos } \\
\text { taurus] }\end{array}$ & $56 \%$ & 74 & 12 & 7 \\
\hline A12 & No Significant hit & & & & \\
\hline B1 & $\begin{array}{l}\text { Alpha-2-macroglobulin precursor } \\
\text { (Alpha-2-M) }\end{array}$ & $42 \%$ & 209 & 49 & 4 \\
\hline B2 & No Significant hit & & & & \\
\hline B3 & $\begin{array}{l}\text { alpha-1 acid glycoprotein [Bos } \\
\text { taurus] }\end{array}$ & $56 \%$ & 91 & $\begin{array}{c}11 \\
0.11 \\
\text { delta }\end{array}$ & 7 \\
\hline B4 & Chain A, Recombinant Avidin & $73 \%$ & 85 & 12 & 4 \\
\hline B5 & No Significant hit & & & & \\
\hline B6 & $\begin{array}{l}\text { Chain A, Crystal Structure } \text { of } \\
\text { Aluminum-Bound Ovotransferrin At } 2.15 \\
\text { Angstrom Resolution }\end{array}$ & $20 \%$ & 79 & 16 & 5 \\
\hline B7 & No Significant hit & & & & \\
\hline B8 & albumin [Bos taurus] & $35 \%$ & 81 & 23 & 11 \\
\hline B9 & No Significant hit & & & & \\
\hline B10 & $\begin{array}{l}\text { dehydrogenase, glyceraldehydephosphate } \\
\text { (pig) Rabbit GAPDH showed non } \\
\text { significant score }\end{array}$ & $38 \%$ & 87 & 11 & 3 \\
\hline B11 & $\begin{array}{l}\text { Chain O, Crystal Structure of The } \\
\text { Rabbit Muscle Glyceraldehyde-3- } \\
\text { Phosphate Dehydrogenase (Gapdh) }\end{array}$ & $42 \%$ & 102 & 15 & 4 \\
\hline B12 & $\begin{array}{l}\text { Chain } \text {, Myoglobin (Horse } \text { Heart) } \\
\text { Mutant With Leu } 104 \text { Replaced By Asn } \\
\text { (L104n) (Insignificant score) }\end{array}$ & $71 \%$ & 76 & 10 & 4 \\
\hline $\mathrm{C} 1$ & Keratin Contamination & & & & \\
\hline $\mathrm{C} 2$ & $\begin{array}{l}\text { actin, alpha 1, skeletal muscle [Bos } \\
\text { taurus] }\end{array}$ & $36 \%$ & 110 & 12 & 2 \\
\hline $\mathrm{C} 3$ & No Significant hit & & & & \\
\hline $\mathrm{C} 4$ & No Significant hit & & & & \\
\hline
\end{tabular}




\begin{tabular}{|c|c|c|c|c|c|}
\hline $\mathrm{C} 5$ & No Significant hit & & & & \\
\hline C6 & albumin [Bos taurus] & $26 \%$ & 91 & 17 & 6 \\
\hline $\mathrm{C} 7$ & $\begin{array}{l}\text { albumin [Bos taurus] (Non significant } \\
\text { hit) }\end{array}$ & $22 \%$ & 55 & 12 & 5 \\
\hline $\mathrm{C} 8$ & $\begin{array}{l}\text { Chain O, Crystal Structure Of The } \\
\text { Rabit Muscle Glyceraldehyde-3- } \\
\text { Phosphate Dehydrogenase (Gapdh) }\end{array}$ & $47 \%$ & 97 & 17 & 6 \\
\hline C9 & $\begin{array}{l}\text { Chain O, Crystal Structure Of The } \\
\text { Rabit Muscle Glyceraldehyde-3- } \\
\text { Phosphate Dehydrogenase (Gapdh) }\end{array}$ & $47 \%$ & 125 & 18 & 7 \\
\hline $\mathrm{C} 10$ & $\begin{array}{llll}\text { Chain } & \text { Myoglobin (Horse } & \text { Heart) } \\
\text { Mutant With Leu } 104 \text { Replaced By Asn } \\
\text { (L104n) (Non significant hit) } & \\
\end{array}$ & $60 \%$ & 70 & 9 & 4 \\
\hline $\mathrm{C} 11$ & $\begin{array}{l}\text { actin, alpha 1, skeletal muscle [Bos } \\
\text { taurus] }\end{array}$ & $40 \%$ & 79 & 14 & 5 \\
\hline $\mathrm{C} 12$ & $\begin{array}{l}\text { actin, alpha 1, skeletal muscle [Bos } \\
\text { taurus] }\end{array}$ & $43 \%$ & 84 & 13 & 3 \\
\hline D1 & $\begin{array}{l}\text { Chain }{ }_{\text {Mol_id: }} \text { 1; Molecule: } \\
\text { Ovotransferrin; Chain: Null; Synonym: } \\
\text { Conalbumin; Heterogen: Iron (F }\end{array}$ & $60 \%$ & 129 & 43 & 17 \\
\hline D2 & $\begin{array}{l}\text { Chain }, \text { Mol_id: } 1 ; \text { Molecule: } \\
\text { Ovotransferrin; Chain: Null; Synonym: } \\
\text { Conalbumin; Heterogen: Iron (F }\end{array}$ & $62 \%$ & 167 & 46 & 19 \\
\hline D3 & albumin [Bos Taurus & $42 \%$ & 80 & 23 & 7 \\
\hline D4 & albumin [Bos taurus] & $53 \%$ & 162 & 38 & 9 \\
\hline D5 & No Significant hit & & & & \\
\hline D6 & Keratin contamination & & & & \\
\hline D7 & $\begin{array}{l}\text { Chain A, Crystal Structure Analysis } \\
\text { of Bovine Carbonic Anhydrase Ii }\end{array}$ & $71 \%$ & 95 & 18 & 4 \\
\hline D8 & Myoglobin (non significant) & $73 \%$ & 57 & 10 & 2 \\
\hline D9 & $\begin{array}{l}\text { actin, alpha 1, skeletal muscle [Bos } \\
\text { taurus] }\end{array}$ & $54 \%$ & 125 & 25 & 8 \\
\hline D10 & Keratin contamination & & & & \\
\hline D11 & $\begin{array}{l}\text { Chain , Mol_id: 1; Molecule: } \\
\text { Ovotransferrin; Chain: Null; Synonym: } \\
\text { Conalbumin; Heterogen: Iron (F }\end{array}$ & $55 \%$ & 181 & 43 & 18 \\
\hline D12 & $\begin{array}{l}\text { Chain }, \text { Mol_id: } 1 ; \text { Molecule: } \\
\text { Ovotransferrin; Chain: Null; Synonym: } \\
\text { Conalbumin; Heterogen: Iron (F }\end{array}$ & $64 \%$ & 204 & 45 & 18 \\
\hline E1 & No Significant hit & & & & \\
\hline E2 & albumin [Bos taurus] & $62 \%$ & 260 & 38 & 12 \\
\hline E3 & $\begin{array}{l}\text { Chain O, Crystal Structure Of The } \\
\text { Rabit Muscle Glyceraldehyde-3- } \\
\text { Phosphate Dehydrogenase (Gapdh }\end{array}$ & $37 \%$ & 131 & 25 & 10 \\
\hline E4 & $\begin{array}{l}\text { Chain O, Crystal Structure Of The } \\
\text { Rabit Muscle Glyceraldehyde-3- } \\
\text { Phosphate Dehydrogenase (Gapdh) (non } \\
\text { significant score) }\end{array}$ & $42 \%$ & 72 & 21 & 7 \\
\hline E5 & carbonic anhydrase II [Bos taurus] & $70 \%$ & 131 & 17 & 3 \\
\hline E6 & $\begin{array}{cccc}\text { Chain } & \text { Myoglobin } \text { (Horse } & \text { Heart) } \\
\text { Mutant With Leu 104 Replaced By Asn } \\
\text { (L104n (non significant score) } & \\
\end{array}$ & $79 \%$ & 75 & 12 & 5 \\
\hline E7 & $\begin{array}{l}\text { actin, alpha 1, skeletal muscle [Bos } \\
\text { taurus] }\end{array}$ & $46 \%$ & 130 & 20 & 5 \\
\hline E8 & $\begin{array}{lll}\text { Chain B, Complex Porcine } & \text { Pancreatic } \\
\text { TrypsinSOYBEAN TRYPSIN } & \text { Inhibitor, }\end{array}$ & $66 \%$ & 73 & 14 & 4 \\
\hline
\end{tabular}




\begin{tabular}{|c|c|c|c|c|c|}
\hline & $\begin{array}{lll}\text { Tetragonal Crystal } & \text { Form } & \text { (non } \\
\text { significant score) } & & \\
\end{array}$ & & & & \\
\hline E9 & $\begin{array}{l}\text { Chain , Mol_id: } 1 ; \text { Molecule: } \\
\text { Ovotransferrin; Chain: Null; Synonym: } \\
\text { Conalbumin; Heterogen: Iron ( }\end{array}$ & $66 \%$ & 226 & 46 & 18 \\
\hline E10 & $\begin{array}{l}\text { Chain , Mol_id: } 1 \text {; Molecule: } \\
\text { Ovotransferrin; Chain: Null; Synonym: } \\
\text { Conalbumin; Heterogen: Iron (F }\end{array}$ & $69 \%$ & 264 & 52 & 19 \\
\hline E11 & albumin [Bos Taurus & $61 \%$ & 180 & 37 & 14 \\
\hline E12 & keratin contamination & & & & \\
\hline F1 & $\begin{array}{l}\text { Chain O, Crystal Structure of The } \\
\text { Rabbit Muscle Glyceraldehyde-3- } \\
\text { Phosphate Dehydrogenase (Gapdh) }\end{array}$ & $53 \%$ & 123 & 26 & 10 \\
\hline $\mathrm{F} 2$ & $\begin{array}{l}\text { glyceraldehyde-3-phosphate } \\
\text { dehydrogenase [Bos taurus] (NO } \\
\text { rabbit) }\end{array}$ & $46 \%$ & 89 & 23 & 8 \\
\hline F3 & No Significant hit & & & & \\
\hline $\mathrm{F} 4$ & No Significant hit & & & & \\
\hline F5 & $\begin{array}{l}\text { actin, alpha } 1 \text {, skeletal muscle [Bos } \\
\text { taurus] }\end{array}$ & $37 \%$ & 82 & 11 & 2 \\
\hline F6 & $\begin{array}{l}\text { Chain B, Complex Porcine Pancreatic } \\
\text { TrypsinSoYBEAN TRYPIN Inhibitor, } \\
\text { Tetragonal Crystal Form }\end{array}$ & $49 \%$ & 90 & 12 & 4 \\
\hline F7 & $\begin{array}{l}\text { Chain } \text { Mol_id: } 1 \text {; Molecule: } \\
\text { Ovotransferrin; Chain: Null; Synonym: } \\
\text { Conalbumin; Heterogen: Iron (F }\end{array}$ & $50 \%$ & 201 & 36 & 13 \\
\hline F8 & $\begin{array}{l}\text { Chain , Mol_id: } 1 \text {; Molecule: } \\
\text { Ovotransferrin; Chain: Null; Synonym: } \\
\text { Conalbumin; Heterogen: Iron (F }\end{array}$ & $67 \%$ & 284 & 52 & 21 \\
\hline F9 & albumin [Bos taurus] & $55 \%$ & 115 & 28 & 11 \\
\hline F10 & albumin [Bos taurus] & $70 \%$ & 168 & 46 & 14 \\
\hline F11 & $\begin{array}{l}\text { dehydrogenase, glyceraldehydephosphate } \\
\text { (No rabbit) }\end{array}$ & $34 \%$ & 92 & 18 & 6 \\
\hline F12 & No significant hit & & & & \\
\hline G1 & $\begin{array}{l}\text { glyceraldehyde-3-phosphate } \\
\text { dehydrogenase [Bos taurus] (No } \\
\text { rabbit) }\end{array}$ & $43 \%$ & 93 & 24 & 9 \\
\hline $\mathrm{G} 2$ & $\begin{array}{llll}\text { Chain } & \text { Myoglobin (Horse } & \text { Heart) } \\
\text { Mutant With Leu } 104 \text { Replaced By Asn } \\
\text { (L104n) (non significant hit) } & \\
\end{array}$ & $65 \%$ & 70 & 9 & 3 \\
\hline G3 & No significant hit & & & & \\
\hline G4 & $\begin{array}{l}\text { Chain B, Complex Porcine } \text { Pancreatic } \\
\text { TrypsinsoyBEAN TRYPIN Inhibitor, } \\
\text { Tetragonal Crystal Form }\end{array}$ & $54 \%$ & 107 & 13 & 5 \\
\hline G5* & $\begin{array}{l}\text { MIXTURE: Chain A, Crystal Structure } \\
\text { Of Aluminum-Bound Ovotransferrin At } \\
2.15 \text { Angstrom Resolution } \\
\text { Chain A, Soybean Trypsin Inhibitor }\end{array}$ & & 209 & & \\
\hline G6 & $\begin{array}{l}\text { Chain , Mol id: } 1 ; \text { Molecule: } \\
\text { Ovotransferrin; Chain: Null; Synonym: } \\
\text { Conalbumin; Heterogen: Iron (F }\end{array}$ & $62 \%$ & 228 & 48 & 17 \\
\hline G7 & albumin [Bos taurus] & $54 \%$ & 120 & 30 & 10 \\
\hline G8 & albumin [Bos taurus] & $\mathrm{Q}$ & 160 & 42 & 21 \\
\hline G9 & $\begin{array}{l}\text { glyceraldehyde-3-phosphate } \\
\text { dehydrogenase [Bos Taurus (NO rabbit) }\end{array}$ & $44 \%$ & 91 & 20 & 7 \\
\hline G10 & Chain 0 , Crystal structure of The & $35 \%$ & 92 & 17 & 6 \\
\hline
\end{tabular}




\begin{tabular}{|c|c|c|c|c|c|}
\hline & $\begin{array}{l}\text { Rabbit Muscle Glyceraldehyde-3- } \\
\text { Phosphate Dehydrogenase (Gapdh) }\end{array}$ & & & & \\
\hline G11 & carbonic anhydrase II [Bos taurus] & $56 \%$ & 100 & 14 & 4 \\
\hline G12 & $\begin{array}{l}\text { Chain } \\
\text { Mutant With Leu 104 } \\
\text { (L104n }\end{array}$ & $81 \%$ & 81 & 13 & 5 \\
\hline H1 & $\begin{array}{l}\text { actin, alpha 1, skeletal muscle [Bos } \\
\text { taurus] }\end{array}$ & $56 \%$ & 119 & 22 & 8 \\
\hline $\mathrm{H} 2$ & $\begin{array}{l}\text { Chain B, Complex Porcine Pancreatic } \\
\text { TrypsinSOYBEAN TRYPSIN Inhibitor, } \\
\text { Tetragonal Crystal Form }\end{array}$ & $54 \%$ & 113 & 12 & 4 \\
\hline H3 & $\begin{array}{l}\text { Chain , Mol_id: } 1 ; \text { Molecule: } \\
\text { Ovotransferrin; Chain: Null; Synonym: } \\
\text { Conalbumin; Heterogen: Iron (F }\end{array}$ & $71 \%$ & 191 & 26 & 60 \\
\hline $\mathrm{H} 4$ & $\begin{array}{l}\text { Chain }, \text { Mol_id: } 1 ; \text { Molecule: } \\
\text { Ovotransferrin; Chain: Null; Synonym: } \\
\text { Conalbumin; Heterogen: Iron (F }\end{array}$ & $41 \%$ & 139 & 27 & 9 \\
\hline $\mathrm{H} 5$ & albumin [Bos taurus] & $62 \%$ & 151 & 37 & 13 \\
\hline H6 & albumin [Bos taurus] & $67 \%$ & 128 & 44 & 19 \\
\hline $\mathrm{H7}$ & No significant hit & & & & \\
\hline H8 & $\begin{array}{l}\text { Chain O, Crystal Structure of The } \\
\text { Rabbit Muscle Glyceraldehyde-3- } \\
\text { Phosphate Dehydrogenase (Gapdh }\end{array}$ & $47 \%$ & 89 & 18 & 4 \\
\hline H9 & $\begin{array}{l}\text { carbonic anhydrase II [Bos taurus] } \\
\text { (Keratin contamination) }\end{array}$ & $77 \%$ & 89 & $\begin{array}{c}19 \\
0.1 \text { delta }\end{array}$ & 5 \\
\hline H10 & Myoglobin (NO significant hit) & $79 \%$ & 70 & 11 & 3 \\
\hline H11 & $\begin{array}{l}\text { actin, alpha 1, skeletal muscle [Bos } \\
\text { taurus] }\end{array}$ & $57 \%$ & 105 & 19 & 6 \\
\hline $\mathrm{H} 12$ & Chain A, Soybean Trypsin Inhibitor & $58 \%$ & 91 & 14 & 4 \\
\hline
\end{tabular}

* Sample G5 contained a mix of two standards.

\section{Statistical analysis.}

All peptides recovered from each of the proteins for all stains were collected from the MASCOT database results (Table S-1). Sequences were examined for amino acid composition with the ProtParam tool (SWISS-Prot) and compared to the theoretical compositions of the proteins determined by in silico digestion (ProtMass; SWISS-Prot).

Compositional analysis was calculated by subtracting the amino acid composition of all the peptides isolated from each stain from the theoretical composition of all the peptides theoretically isolatable by in silico digestion allowing for up to 1 missed cleavage. This numberwas divided by the theoretical composition and expressed as a percentage (Table S-2). Positive numbers indicate relative loss of that amino acid and negative numbers indicate an over abundance of that amino acid.

This number can also be expressed as proportion of the total peptides recovered - and this value is directly comparable to the theoretical recovery of peptides, a two population test of proportions was used to determine if the observed differences in peptides recovered from each stain was significantly different with what would be expected from in silico digestion 
The test of two population proportions is given by:

$$
z=\frac{\left(\hat{p}_{1}-\hat{p}_{2}\right)-\left(\bar{p}_{1}-\bar{p}_{2}\right)}{\sqrt{\bar{p}(1-\bar{p})\left(\frac{1}{n_{1}}+\frac{1}{n_{2}}\right)}}
$$

Where: $n=$ number of peptides in each sample

$\mathrm{p}($ hat $)=$ the proportion of the population that contains the amino acid being tested $\mathrm{p}($ bar $)=\left(\mathrm{p}(\text { hat })_{1} \times \mathrm{n}_{1}+\mathrm{p}(\text { hat })_{2} \times \mathrm{n}_{2}\right) /\left(\mathrm{n}_{1}+\mathrm{n}_{2}\right)$

The confidence value $(p)$ is calculated as $2 \times(1$-normal distribution of $z)$

Table S-2. In silico amino acid composition of all recovered peptides compared to the theoretical composition of all the proteins in the 2D sample. Deviation greater than $25 \%$ are highlighted.

\begin{tabular}{|l||r|r|r|r|}
\hline Amino Acid & \multicolumn{1}{|c|}{ SR (\%) } & \multicolumn{1}{|c|}{ LP (\%) } & \multicolumn{1}{|c|}{ FL (\%) } & \multicolumn{1}{c|}{ KR (\%) } \\
\hline Ala (A) & -12.66 & -3.80 & 2.53 & 0.00 \\
\hline Arg (R) & -10.00 & -7.50 & -5.00 & 5.00 \\
\hline Asn (N) & 8.11 & -24.32 & -18.92 & -16.22 \\
\hline Asp (D) & 18.18 & -6.06 & -4.55 & 0.00 \\
\hline Cys (C) & 63.33 & -16.67 & -36.67 & -30.00 \\
\hline GIn (Q) & 18.75 & 6.25 & 15.63 & 15.63 \\
\hline Glu (E) & -10.00 & 0.00 & -8.57 & -10.00 \\
\hline Gly (G) & -4.35 & -2.90 & 11.59 & 2.90 \\
\hline His (H) & -32.14 & -3.57 & 0.00 & -28.57 \\
\hline Ile (I) & -4.35 & 21.74 & 13.04 & 21.74 \\
\hline Leu (L) & 11.90 & 2.38 & 7.14 & 0.00 \\
\hline Lys (K) & 22.22 & 24.69 & 14.81 & 14.81 \\
\hline Met (M) & -77.78 & -27.78 & -22.22 & -16.67 \\
\hline Phe (F) & -9.52 & 4.76 & -9.52 & -2.38 \\
\hline Pro (P) & -21.74 & -4.35 & -2.17 & -6.52 \\
\hline Ser (S) & 16.67 & 1.67 & 0.00 & 3.33 \\
\hline Thr (T) & -7.14 & -1.79 & -1.79 & 0.00 \\
\hline Trp (W) & 75.00 & 25.00 & 16.67 & 16.67 \\
\hline Tyr (Y) & 0.00 & -6.45 & 0.00 & -3.23 \\
\hline Val (V) & -29.58 & -7.04 & -7.04 & 1.41 \\
\hline
\end{tabular}

* variation from theoretical amino acid composition (Theoretical - Observed)*100/Theoretical 
Table S-3. Two population test for cysteine containing peptides.

\begin{tabular}{|c|c|c|c|c|c|}
\hline \multicolumn{3}{|c|}{ SYPRO Ruby vs Theoretical values } & \multicolumn{3}{|c|}{ LavaPurple vs Theoretical values } \\
\hline & Theoretical & SYPRO Ruby & & IvaPurple & Theoretical \\
\hline $\mathbf{n}=$ & 200 & 166 & $\mathbf{n}=$ & 396 & 487 \\
\hline$p$ hat $=$ & 0.29 & 0.09 & $p$ hat $=$ & 0.343 & 0.335 \\
\hline p-bar $=$ & 0.19929 & & p-bar = & 0.338588 & \\
\hline bottom line = & 0.041942 & & bottom line $=$ & 0.032021 & \\
\hline top line = & 0.2 & & top line $=$ & 0.008 & \\
\hline$z=$ & 4.768463 & & $z=$ & 0.249833 & \\
\hline$p$-value $=$ & 0.000002 & & $p$-value $=$ & 0.802717 & \\
\hline Flamingo vs Th & oretical values & & Krypton vs Th & oretical va & ues \\
\hline & Flamingo & Theoretical & & Krypton & Theoretical \\
\hline$n=$ & 408 & 487 & $\mathbf{n}=$ & 393 & 487 \\
\hline$p$ hat $=$ & 0.377 & 0.335 & $p$ hat $=$ & 0.389 & 0.335 \\
\hline p-bar $=$ & 0.35414637 & & p-bar $=$ & 0.359116 & \\
\hline bottom line = & 0.03209781 & & bottom line $=$ & 0.03253 & \\
\hline top line $=$ & 0.042 & & top line $=$ & 0.054 & \\
\hline$z=$ & 1.30850048 & & $z=$ & 1.659992 & \\
\hline$p$-value $=$ & 0.190704 & & $p$-value $=$ & 0.096916 & \\
\hline
\end{tabular}

Table S-4. Two population test for histidine containing peptides.

\begin{tabular}{|c|c|c|c|c|c|}
\hline \multicolumn{3}{|c|}{ SYPRO Ruby vs Theoretical values } & \multicolumn{3}{|c|}{ LavaPurple vs Theoretical values } \\
\hline & SYPRO Ruby & Theoretical & & LavaPurple & Theoretical \\
\hline $\mathbf{n}=$ & 166 & 487 & $\mathrm{n}=$ & 419 & 487 \\
\hline p hat $=$ & 0.428 & 0.314 & $\mathrm{p}$ hat $=$ & 0.351 & 0.314 \\
\hline p-bar $=$ & 0.34298 & & p-bar $=$ & 0.331111 & \\
\hline & & & bottom line & & \\
\hline bottom line $=$ & 0.042664 & & $=$ & 0.031359 & \\
\hline top line $=$ & 0.114 & & top line $=$ & 0.037 & \\
\hline$z=$ & 2.672041 & & $z=$ & 1.1799 & \\
\hline$p$-value $=$ & 0.007539 & & p-value $=$ & 0.238040 & \\
\hline
\end{tabular}

Flamingo vs Theoretical values

Krypton vs Theoretical values

$\begin{array}{rrr} & \text { Flamingo } & \text { Theoretical } \\ \mathbf{n}= & 433 & 487 \\ \mathbf{p} \text { hat }= & 0.358 & 0.314 \\ \mathbf{p} \text {-bar }= & 0.334709 & \end{array}$

$\begin{array}{rrr} & \text { Krypton } & \text { Theoretical } \\ \mathbf{n}= & 433 & 487 \\ \mathbf{p ~ h a t ~}= & 0.436 & 0.314 \\ \mathbf{p} \text {-bar }= & 0.37142 & \end{array}$




$$
\begin{array}{rrrr}
\text { bottom line }= & 0.031169 & \text { bottom line }= & 0.031915 \\
\text { top line }= & 0.044 & \text { top line }= & 0.122 \\
\mathbf{z}= & 1.411652 & \mathbf{z}= & 3.822624 \\
\mathbf{p} \text {-value }= & 0.158052 & \mathbf{p} \text {-value }= & 0.000132
\end{array}
$$

\begin{tabular}{|c|c|c|}
\hline \multicolumn{3}{|c|}{ SYPRO Ruby vs Theoretical values } \\
\hline & SYPRO Ruby & Theoretic \\
\hline $\mathrm{n}=$ & 166 & \\
\hline p hat $=$ & 0.319 & 0.2 \\
\hline p-bar $=$ & 0.232489 & \\
\hline bottom line $=$ & 0.037965 & \\
\hline top line = & 0.116 & \\
\hline$z=$ & 3.055461 & \\
\hline$p$-value $=$ & 0.002247 & \\
\hline Flamingo vs $\mathrm{Tr}$ & eoretical values & \\
\hline & Flamingo Th & leoretical \\
\hline $\mathrm{n}=$ & 433 & 487 \\
\hline$p$ hat $=$ & 0.217 & 0.203 \\
\hline p-bar = & 0.209589 & \\
\hline bottom line = & 0.026884 & \\
\hline top line = & 0.014 & \\
\hline$z=$ & 0.520753 & \\
\hline p-value $=$ & 0.602539 & \\
\hline
\end{tabular}

Table S-5. Two population test for methionine containing peptides:

LavaPurple vs Theoretical values 487

\begin{tabular}{|c|c|c|}
\hline LavaPt & Theoretical va & ue \\
\hline & Theoretical & LavaPurple \\
\hline $\mathbf{n}=$ & 487 & 41 \\
\hline p hat $=$ & 0.154 & 0.129 \\
\hline p-bar $=$ & 0.142438 & \\
\hline
\end{tabular}

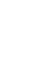

$$
\begin{array}{rr}
\text { bottom line }= & 0.028014 \\
\text { top line }= & 0.057 \\
\mathbf{z}= & 2.034684 \\
\mathbf{p} \text {-value }= & 0.041883
\end{array}
$$

Krypton vs Theretical values

$$
\begin{array}{rrr}
\mathbf{n}= & \text { Krypton } & \text { Theoretical } \\
\mathbf{p} \text { hat }= & 433 & 487 \\
\mathbf{p} \text {-bar }= & 0.256 & 0.203 \\
& & \\
\text { bottom line }= & 0.027709 \\
\text { top line }= & 0.053 \\
\mathbf{z}= & 1.912721 \\
\mathbf{p} \text {-value }= & 0.055784
\end{array}
$$

Table S-6. Two population test for tryptophan containing peptides.

$\begin{array}{rrr}\text { SYPRO Ruby vs Theoretical values } \\ \text { Theoretical } & \text { SYPRO Ruby } \\ \mathbf{n}= & 200 & 166 \\ \mathbf{p} \text { hat }= & 0.13 & 0.048 \\ \mathbf{p} \text {-bar }= & 0.092809 & \end{array}$

$$
\begin{array}{rr}
\text { bottom line }= & 0.030466 \\
\text { top line }= & 0.082 \\
\mathbf{z}= & 2.691525 \\
\mathbf{p} \text {-value }= & 0.007113
\end{array}
$$

$$
\begin{array}{rr}
\text { bottom line }= & 0.023288 \\
\text { top line }= & 0.025 \\
\mathbf{z}= & 1.073499
\end{array}
$$$$
\text { p-value }=\quad 0.283048
$$ 
Flamingo vs Theoretical values

$\begin{array}{rrr}\mathbf{n}= & \text { Theoretical } & \text { Flamingo } \\ \mathbf{p} \text { hat }= & 487 & 433 \\ \mathbf{p} \text {-bar }= & 0.154 & 0.125 \\ & 0.140351 & \\ \text { bottom line }= & 0.022943 \\ \text { top line }= & 0.029 \\ \mathbf{z}= & 1.263991 \\ \mathbf{p} \text {-value }= & 0.206233\end{array}$

$\begin{array}{rrr}\text { Krypton vs Theoretical values } \\ \mathbf{n}= & \text { Theoretical } & \text { Krypton } \\ \mathbf{p} \text { hat }= & 487 & 433 \\ \mathbf{p} \text {-bar }= & 0.154 & 0.151 \\ & 0.152588 & \\ \text { bottom line }= & 0.023752 & \\ \text { top line }= & 0.003 & \\ \mathbf{z}= & 0.126307 \\ \mathbf{p} \text {-value }= & 0.899489\end{array}$

Table S-7. Two population test for valine containing peptides.

SYPRO Ruby vs Theoretical values

$\begin{array}{rrr} & \text { SYPRO Ruby } & \text { Theoretical } \\ \mathbf{n}= & 166 & 487 \\ \mathbf{p} \text { hat }= & 0.627 & 0.563 \\ \mathbf{p} \text {-bar }= & 0.57927 & \\ & & \\ \text { bottom line }= & 0.044369 & \\ \text { top line }= & 0.064 & \\ \mathbf{z}= & 1.442446 & \\ \mathbf{p} \text {-value }= & 0.149177\end{array}$

Flamingo vs Theoretical values

$\begin{array}{rrr}\mathbf{n}= & \text { Theoretical } & \text { Flamingo } \\ \mathbf{p} \text { hat }= & 487 & 433 \\ \mathbf{p} \text {-bar }= & 0.56017609 & 0.557 \\ & \\ \text { bottom line }= & 0.03278591 & \\ \text { top line }= & 0.006 & \\ \mathbf{z}= & 0.18300547 \\ \mathbf{p} \text {-value }= & 0.854794\end{array}$

LavaPurple vs Theoretical values

$\begin{array}{rrr}\mathbf{n}= & \text { Theoretical } & \text { LavaPurple } \\ \mathbf{p} \text { hat }= & 487 & 419 \\ \mathbf{p} \text {-bar }= & 0.553288 & 0.542 \\ & & \\ \text { bottom line }= & 0.033127 \\ \text { top line }= & 0.021 & \\ \mathbf{z}= & 0.633924 \\ \mathbf{p} \text {-value }= & 0.526130\end{array}$

Krypton vs Theoretical values

$\begin{array}{rrr}\mathbf{n}= & \text { Theoretical } & \text { Krypton } \\ \mathbf{p} \text { hat }= & 487 & 433 \\ \mathbf{p} \text {-bar }= & 0.563 & 0.542 \\ & & \\ \text { bottom line } & & \\ = & 0.0353116 & \\ \text { top line }= & 0.021 & \\ \mathbf{z}= & 0.639482 \\ \mathbf{p} \text {-value }= & 0.522509\end{array}$

Therefore none of the stains recovers significantly different levels of peptides containing valine even though the compositional analysis suggests that this is the case. Closer examination of the peptides recovered indicates a large number have multiple valines per peptide and were associated with methionine containing peptides so the increase of this particular amino acid may be artefactual. 